\title{
Is Complexity Theory Useful in Describing Classroom Learning?
}

\author{
Mark Hardman \\ Canterbury Christ Church University, UK \\ mark.hardman@canterbury.ac.uk
}

Presented at:

The European Conference on Educational Research

Helsinki

$2^{\text {th }}$ August 2010 
Within educational research it has become commonplace to refer to the complexity of the situation being investigated. Indeed, the theme of most recent American Educational Research Association (AERA) conference was "Understanding Complex Ecologies in a Changing World". This is undoubtedly because it is recognised that education is a field within which there are a multitude of interlinked factors influencing each situation. However, these references to complexity are increasingly the aligning of an author's discussion with a specific field of thought, rather than the everyday use of the term. Is this continual reference to what is known as complexity theory or complexity science a passing trend or something more tangible and useful?

It is well established that complexity theory first developed within the domain of pure mathematics and was then applied within the natural sciences (McMillan, 2008; Stacey, 2003). In the last decade or so however the social sciences have increasingly adopted and adapted complexity theory to describe social systems. In this paper I wish to explore the contemporary usage of complexity theory in relation to education and in doing so ascertain its usefulness for describing the learning that takes place in classrooms. First I shall outline what complexity theory is within the physical sciences and then use this to investigate what it may mean within education.

In order to understand complexity theory within the natural sciences it is helpful to contrast it to linear science, which was developed within the classical, Newtonian paradigm. Within classical science an input variable is related to an output variable such that the relationship between them can be investigated. This can be referred to as a 'linear' relationship in that there is direct correspondence between input and output. The classical scientific process involves isolating all other variables so that the linear relationship may be investigated and from this equations are usually developed which describe the behaviour of the system. In the mathematical sense these equations may still contain 'nonlinear' terms (e.g. $a^{2}$ or ab) but nevertheless describe the relationship between two variables only and thus may be denoted linear relationships. These equations can be used to predict the development of the system given any starting point and therefore the 'history' of the system is not important: if we know the starting conditions we know the future of the system. An example of a linear system is a simple pendulum, for which simple equations are able to determine the position, speed and acceleration of the pendulum for a given time after it is released.

In the early part of the $20^{\text {th }}$ century scientists believed that this scientific method of isolating variables would be able to describe the workings of the whole universe. By the end of the $20^{\text {th }}$ century however this dream was abandoned. It became apparent that the situations for which variables can be neatly isolated are not the norm but exceptions. The majority of real situations involve 'nonlinear' relationships. That is, the variables cannot be isolated and investigated independently but must be recognised as mutually dependent. With the growth of chaos theory it was found that many of these nonlinear systems are highly sensitive to initial conditions. In the case of two pendula linked together it can be seen ${ }^{1}$ that the motion quickly becomes seemingly unpredictable, and furthermore that the motion is extremely sensitive to the initial conditions. The history of the system is important and no longer can we describe the motion as a function of time alone.

\footnotetext{
${ }^{1}$ I shall be demonstrating both a simple and double pendulum.
} 
The double pendulum can actually still be described by equations of motion and as such can be labelled as chaotic. Conversely, a complex system is one in which there are a large number of variables interacting without any overall equation determining their motion. The subtle yet important difference between a chaotic and complex system is that a chaotic system is deterministic, whereas the development of a complex system is indeterminate. All real systems are indeterminate (Prigogine, 1997). Whilst the mathematical description of the double pendulum is chaotic, the interaction of this system with the air around it and the internal friction of the system mean that in reality it is a complex system.

Clearer distinction between chaotic and complex systems comes from models of multiple agents such as the flocking or birds or the foraging of ants'. In the case of bird flocking Wilensky's (1998) model shows that birds may form flocks by adhering to only three simple rules. Firstly that they tend to turn to fly in the same direction; secondly that they will avoid colliding through getting too close; but thirdly that they will tend to move towards each other (up to the limit of being too close). In Wilensky's (1997) model of ant behaviour we see that ants initially move randomly. Once they find food they return to the nest and release a chemical pheromone. Other ants nearby follow this pheromone and in this way the food is quickly collected by a line of ants. Both of these models typify complex systems in which a large number of agents respond only to simple rules in their immediate environment yet there is emergent behaviour in the system.

But what has all this got to do with education? Well, to illustrate the insight complexity theory may provide, let us return to our descriptions of linear and non-linear science. With respect to a linear formulation consider the UK Office for Standards in Education report "Using Data Improving Schools" (Ofsted, 2008). In this document Ofsted illustrates the correspondence between test results for 11 year olds and exam results for 16 year olds. Schooling here is being conceived of as a linear process which relates input directly to output in clear analogy to a classical science viewpoint. By returning to our consideration of complexity however, we are able to suggest that the schooling system is not that simple. Experience suggests that a multitude of factors involved in schooling including curriculum, parents, teaching contexts, classmates and even such seemingly irrelevant factors as the weather outside do have influence upon the system. Complexity theory gives us a lens through which we can appreciate the interaction of all these factors and describe education as dynamic and highly sensitive.

Of course, this is quite a speculative example that doesn't stand much scrutiny. However, there is a broad range of educational fields where complexity theory is being applied. Osberg (2005), Doll (2008) and Mason (2008) have applied complexity theory to understanding how learning emerges from curricula. Radford (2006), and Phelps and Graham (2010) have applied complexity to action research and specifically its limitations. Cilliers (1998) and Kelso (1995) have applied complexity to looking at the neurology of learning. Van Geert (2000) and Smith and Thelen (2003) have developed models of learning through the lens of complex adaptive systems. Davis and Sumara (2006) and Sullivan (2009) use complexity to describe learning in the classroom and I would furthermore situate my own research within this field.

Clearly there must be some attraction and benefit to using complexity theory. Despite this range of applications however, there is still considerable difficultly in defining complexity within education

\footnotetext{
${ }^{2}$ I shall be demonstrating models taken from http://ccl.northwestern.edu/netlogo/models/
} 
and in deciding how it should be used. This is in no small part due to the resistance of complex systems to a unified description: they are all different and constantly developing. There is a dire need to try and overcome this difficulty and analyse current usage to see if any commonality can be found, or to explain why it cannot. Furthermore it is necessary to establish the usefulness of applying concepts from the natural sciences to education. I believe too many authors have 'borrowed' concepts from other fields without considering the impact of this transfer between disciplines and this is certainly true of complexity theory.

In order to analyse current usage of complexity theory I applied a methodological framework comprising three elements. Firstly, I considered Richardson \& Cilliers' (2001) analysis of the state of complexity science at the turn of the century. They suggested that there were three broad approaches to complexity: hard complexity science; soft complexity science and complexity thinking. I shall discuss each of these in turn shortly, whilst also describing how I believe these approaches have changed over the last decade. Secondly, my analytical framework consists of analysing what has changed in bringing concepts from the natural sciences into the field of education. Whilst some would argue that I am trying to hold on to a scientific epistemology in relating complexity to empirical evidence, I stand by this methodology. If we are to move complexity from the empirical foundations of the physical sciences we must see what is left to support it. It is important to see how meanings shift when used in different fields, not only to allow complexity theorists within different fields to communicate but more importantly to identify where authors are actually proposing new concepts. It is unacceptable to characterise a system purely through analogy to flocks of birds or nests of ants without any examination of the system being described. In order to aid analysis of this shifting terminology I classified changes of concepts between fields as additions: where new concepts had been added which are not present in the natural sciences; misunderstanding: where terms from the natural sciences are applied loosely or incorrectly and reinterpretation: where concepts take on a whole new meaning in different fields. The third element of my analysis was to focus primarily on educational literature and thus depart from the broader analysis conducted by Richardson \& Cilliers (2001).

The most straightforward of the three approaches to complexity science identified by Richardson and Cilliers (2001) to deal with is what they call hard complexity science. Hard complexity scientists continue to follow the modernist (or structuralist) approach of seeking universal laws with which they can explain complex systems. Often they build computational models in order to discover the underlying principles of a phenomenon. I would argue that what they actually find is the underlying principles of the model they have built, rather than the phenomenon itself. Whilst this approach has been applied to economics (and we are all aware of the recent failures of economic modelling) it is rarely seen within the social sciences and I have never seen it applied to education. Perhaps social scientists are intrinsically aware of the impossibility of describing human action through equations alone. There is a greater rebuttal of this approach than simply its difficulty however. Complexity theory itself suggests that in a truly complex system the sensitivity of interacting elements means that any approximation of the system will result in the model behaving significantly different to the original system. Complex systems are resistant to reduction; that is what makes them complex and also what renders the approach of hard complexity science untenable.

Soft complexity science is an approach that recognises complexity as a metaphor for social systems. Those who take this approach recognise that the social world is intrinsically different from the 
natural world and that language and meaning are important aspects which are not accounted for in science. This approach is particularly dominant within management and organisational sciences and is increasingly being applied to school leadership (Morrison, 2002). It is also used as the basis for building models of how people learn, although the focus of these models on specific parameters means the problem of reduction is diminished (although not avoided). For example, Smith and Thelen (2003) build on Kelso's (2000) approach by analysing the movement of a child as they learn to perform a task. In this way the dynamics of learning might be investigated through observing events such as when a child reaches for a toy or how their legs move as they learn to walk. Smith and Thelen focus on one parameter and use it to investigate the dynamics of the system but in doing so accept that this will reveal only part of the picture and that the complexity of learning cannot be fully understood in this way.

Whereas Smith and Thelen are clear in how they define their system however, others are not so clear and this is problematic. There are a number of risks associated with drawing on metaphor alone. Firstly there is a likelihood of applying metaphor for metaphor's sake. Without any attempt at definition, it is virtually impossible to judge the usefulness of applying complexity theory to understanding a situation. It is no more clear that phenomena are caused by the interaction of elements within a complex system than if we were to claim that they are being caused by good and evil spirits. Furthermore it is not clear whether the appeal to analogy between social systems and physical systems adds any new insight beyond what could be seen through other social scientific approaches. Does complexity tell us anything new?

One possible new insight comes from the reference to complex systems being at the 'edge of chaos (Davies, 2004; Davis and Sumara (2006)). This concept recognises that in the physical sciences a system is able to maintain itself through adaptation to the environment such that it is 'stable' yet dynamic. If a specific parameter is changed beyond this stable range the system may become unstable and then an increasing number of different developments are possible. In a physical system the plot of some parameter, such as temperature or concentration, shows that the possible paths available to the system expand exponentially past a critical point. This exponential increase is a characteristic of mathematical descriptions of chaos and as such the critical point might be called the 'edge of chaos'. In the social sciences literature this is taken to mean that a social system is constantly adaptive and that the system should be maintained in a state at the edge of chaos in order that it can adapt according to the environment.

At first glance this seems reasonable, but the difficulty comes when we try to describe what we mean by chaos in social systems, or when we try to determine what parameter or characteristic might signify this transition to chaos. For the most part, the term chaos is taken to mean the complete disorder of a system, but I have never seen a satisfactory description of what a completely disordered social system looks like. More careful authors often discuss the mathematical origins of the term chaos and note that it is not a state of randomness but a mathematically determined change in a system. Furthermore they claim it may be seen as a positive thing in which individuals might affect large scale change (Davies, 2004; Lewin, 1993). Again, this seems reasonable. The issue is that because there is no definition of parameters with which to describe a social system, there is a paradox in whether a system is at the edge of chaos or simply chaotic. A physical system is either stable or undergoing change (what scientists call bifurcation). So which is it? If social systems are constantly changing they have chaotic trajectories, so they are not at the edge of chaos at all. This 
issue will only be solved with clearer discussion of what chaos means in social systems. Prigogine (1997) described the mechanisms by which physical systems change from one stable state to another and in doing so allowed complex systems to be understood. Until we are able to do this with social systems we are trapped in the realm of speculation through analogy. Discussion of information flow or conflict of various kinds is present in the literature but this has not yet led to anybody being brave enough to suggest a mechanism for transforming social systems. Gladwell's (2000) book 'the Tipping Point' is perhaps the most famous discussion of mechanisms for sudden social change (bifurcation) but seems to add nothing to this understanding beyond observation that the phenomenon exists.

Part of the issue with uncovering a mechanism for bifurcation within a social system is the fact that humans are aware, at least in some part, of the system they inhabit. All physical and computational descriptions of complex systems assume that 'agents' are only aware of their immediate surroundings. Whilst a modification to how we define an individual's surrounds might allow us to include all the information they receive, the metaphor between physical and human systems will always be stretched to extremes if we appreciate that humans consciously change their social systems, very deliberately, though the use of some understanding of the system as a whole. This difference is telling in that we must characterise complexity differently in physical and social systems and in doing so complexity takes on a new dimension in the social sciences. As such we need to first recognise the differences between the scientific fields in which complexity was developed and the social situations we wish to describe and carefully establish the meaning of terms and concepts in this new field. This is some undertaking indeed.

Perhaps the more immediately tangible insights come from applying complexity not to the descriptions of systems but to the limitations of our understanding. Whereas social scientists have previously recognised that their models and descriptions are limited, complexity science now offers an explanation as to why. The interaction of humans with each other and the environment leads to unpredictable outcomes which are due to the complexity of the system not just a deficiency in the descriptions of it. Analogy to physical systems suggests that we can never truly understand a social system. Whilst this insight might seem to be negative in showing the futility of attempts to fully understand and control social systems, I believe it would actually be welcomed by researchers and teachers alike. Indeed a small scale research project I did discussing this with student teachers suggested that complexity theory gave them a reason for the unpredictability and 'messiness' of classrooms (Hardman, 2010).

This positive take on the limitations of understanding complex systems characterises the current status of what Richardson and Cilliers (2001) denoted complexity thinking. Whilst Richardson and Cilliers (2001) originally conceived of complexity thinking as recognition of "the limited and provisional nature of all understanding" this did not provide much detail of what it entails. They also noted that this form was the least well represented in widespread literature. Almost a decade later however Davis and Sumara (2006) have built directly on this formulation of complexity thinking and have established a school of thought which I would argue is now the dominant form of complexity theory within education in the United States of America. 
Complexity thinking within education today discusses the effectiveness of changing complex systems through recognising the system as thus and creating conditions such that a better system may emerge. 'Emergence' itself has become a key word of this school of thought and recognises the complicit nature of educational practitioners and researchers in affecting the dynamic system. With respect to the classroom this school argues that effective teachers recognise, implicitly at least, that they are functioning in a set of 'nested' complex systems and are able to 'level jump' in dealing with

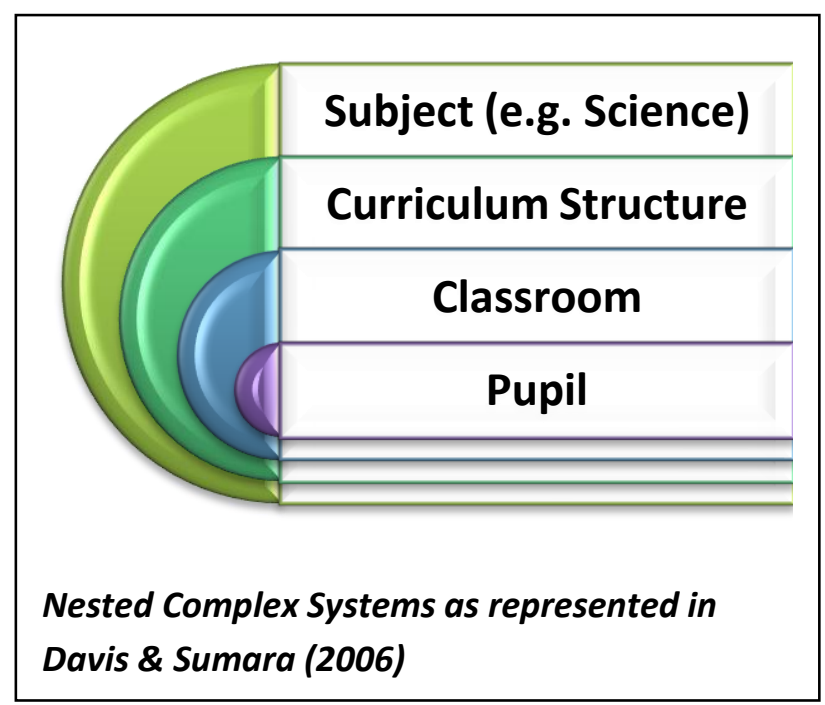
several of these systems at the same time.

This is a positive outlook because it claims that teachers are able to positively influence a complex system such that learning is emergent both on the individual level and in terms of the interaction of individuals - the system itself 'learns'.

For me this is also the source of my two main concerns with complexity thinking. Firstly, we have no way of predicting how a system will develop so although we might say that an individual can influence a system, or nest of

systems, there is no reason to assume that this influence will be a positive one.

Related to this, my second concern is that there is no way of judging the success of action within a complex system without appeal to measures which complexity thinking itself recognises as limited. The approach of many authors is to critique the need for empirical foundation to complex systems as inappropriate for social systems and claim a broader appreciation of epistemology is required in order to judge descriptions of social systems. Whilst in principle this is reasonable, in practice it leads to people claiming that almost everything is a complex system without any tangible reason for characterising it as such.

Beyond another case of metaphor for metaphor's sake however this seems to have a deeper root. Davis and Sumara (2006) align themselves with both Derrida and Foucault in rejecting simple descriptions of a system and in recognising the creation of meaning through the dynamic interaction of humans. What I believe is new within the complexity thinking outlook is the notion that individuals can positively affect meaning within such dynamic systems. Whilst Richardson and Cilliers (2001) and Davis and Sumara (2006) actively try and distance complexity from the trappings of postmodernist philosophy it is difficult to see how complexity thinking can assign merit to any view of the world under the recognition of the dynamic and shifting nature of social systems. Furthermore whilst the positive tendency towards action within complexity thinking is certainly more appealing than most postmodernist positions, it opens practices such as 'level jumping' up to the charge of relativism. If there is no basis on which to judge an action and no way to predict or possibly even describe what the outcome of that action will be then the claim that something positive and useful will emerge seems misguided at best.

Having looked at the three main types of complexity theory within contemporary education let us now review the key points from the literary analysis in order to establish its usefulness to education. 
The lens of complexity theory, of any form, suggests that any representation of a system must be a reduction. When we recognise that humans have individual histories and that their behaviour is based on not only their immediate surrounds but also conscious awareness, we must concede that to describe social systems as complex we are moving beyond descriptions of complex systems in the natural sciences. For this reason social systems are implicitly different from physical systems.

In order to deal with this difference however, which is well recognised, there is a tendency to apply metaphor or analogy to explaining social phenomenon. This too often replaces any real analysis of the specific system being described. We desperately need to move away from metaphor and be able to describe a social system as itself complex. To do this though there must be a focused and concerted effort to define what complexity means in social systems and identify mechanisms for change within those systems. As we have established that every system is different this definition and analysis of mechanism must take place with reference to a specific system and then be done each time we look at a new system.

Only once a detailed description of what constitutes a complex system has been developed can we find a way to judge the effectiveness or otherwise of action within that system. Relying on the assumption that good things will emerge given the right conditions is not sufficient.

This paper set out to look at the usefulness of applying complexity theory to classrooms in particular. I feel that there is simply no way to answer that question without an attempt to describe a classroom, what makes it complex and how it responds to change in its environment. That is the focus of my research currently.

Indications from the review of literature described here are that complexity might have three possible uses. Firstly if a classroom can be described as a complex system then it will be useful in showing that classrooms are sensitive, dynamic and resistant to accurate description. This certainly seems to fit the experience of teachers and would be useful for new teachers to appreciate. Furthermore researchers and policy makers would do well to appreciate the limitations of their insights and allow teachers to operate using their professional experience and judgement.

Secondly then, if a description of a classroom can be developed that is able to define it as complex then further research could focus on how teachers deal with this complexity. Whether appeal to 'level jumping' is enough or whether we need to completely rethink how successful teachers operate is still to be seen. Addressing questions of how teachers learn to teach and go on to become successful teachers is certainly a useful application and the framework of complexity may support valuable insights.

The third and perhaps most optimistic use of complexity would be the development of a description that shows the dynamic sensitivities of classrooms. Whilst being cautious of assuming things will necessarily turn out for the better, it may be possible to describe how teachers control classrooms when they don't want rapid change to the system, and allow it when they do.

All of these applications however rely on first focusing on a classroom and carefully building a description of how interaction of individuals with each other and the environment can be described as a complex system. This must be the next step in evaluating the usefulness of complexity theory to understanding classrooms. 
Cilliers, P. (1998) Complexity \& Postmodernism - Understanding Complex Systems. London:

Routledge

Davis, B. and Sumara, D. (2006) Complexity and education: Inquiries into learning, teaching and research. New Jersey: Lawrence Erlbaum Associates.

Doll, W. (2008) Complexity and the Culture of the Curriculum. Educational Philosophy and Theory, Vol. 40 , No. 1

Gladwell, M. (2000) The Tipping Point. Little, Brown.

Hardman (2010) Learning to Teach in 'Urban Complex Schools'. Conference paper to be presented at British Educational Research Conference, $4^{\text {th }}$ September, Warwick, UK.

Kelso, J.A.S (1995) Dynamic Patterns: The self-Organisation of Brain and Behaviour. Cambridge, Massachusetts: MIT Press

Kelso, J.A.S. (2000) Principles of dynamic pattern formation and change for a science of human behavior. In Developmental Science and the Holistic Approach (Bergman, L.R. and Cairns, R.B., eds), pp. 63-83, Erlbaum

Lewin, R. (1993) Complexity - Life At The Edge Of Chaos. London: Phoenix.

Mason, M. (2008) What is Complexity Theory and What Are Its Implications for Educational Change? Educational Philosophy and Theory, 40 (1) pp35-49

McMillan, E. (2008) Complexity, Management and the Dynamics of Change, London: Routledge

Morrison, K. (2002) School Leadership and Complexity Theory, Routledge Falmer

Ofsted (2008) Using Data Improving Schools

Osberg, D. C. (2005) Curriculum, Complexity and Representation - Rethinking the Epistemology of Schooling Through Complexity Theory. Ph.D Thesis: Open University, UK

Phelps, R \& Graham, A (2010) Exploring the complementarities between complexity and action research: the story of Technology Together', Cambridge Journal of Education, vol. 40, no. 2, pp. 183197.

Prigogine, I. (1997) The end of Certainty: Time, Chaos, and the New Laws of Nature. New York: The Free Press

Prigogine, I. \& Stengers, I. (1989) Order out of Chaos: Man's New Dialogue with Nature. London: HarperCollins

Radford, M. (2006) Researching Classrooms: Complexity \& Chaos. British Educational Research Journal, Vol. 32, No. 2 172-90 
Richardson, K. \& Cilliers, P. (2001) "What is Complexity Science? A View from Different Directions" Emergence, 3(1), p. 5-23

Smith, L.B. and Thelen, E. (2003) Development as a dynamic system. Trends in Cognitive Sciences. Volume 7, Issue 8, August 2003, Pages 343-348

Stacey, R (2003) Complexity and Group Processes. New York: Brunner-Routledge

Sullivan, J. P. (2009) Emergent Learning: The Power of Complex Adaptive Systems in the Classroom. Proceedings of The Complexity and Research in Teacher Education Conference, University of Aberdeen, January 2009. [Available at http://www.abdn.ac.uk/stne/]

van Geert, P. (2000) The dynamics of general developmental mechanisms: from Piaget and Vygotsky to dynamic systems models. Curr. Dir. Psychol. Sci. 9, 64-68

Wilensky, U. (1997). NetLogo Ants model. http://ccl.northwestern.edu/netlogo/models/Ants. Center for Connected Learning and Computer-Based Modeling, Northwestern University, Evanston, IL.

Wilensky, U. (1998). NetLogo Flocking model. http://ccl.northwestern.edu/netlogo/models/Flocking. Center for Connected Learning and Computer-Based Modeling, Northwestern University, Evanston, IL. 